\title{
Optimal siting and sizing of multiple type DGs for the performance enhancement of Distribution System using Differential Evolution Algorithm
}

\author{
N. Karuppiaha ${ }^{\mathrm{a}}$, S. Muthubalaji ${ }^{\mathrm{b}}$, J.Shanmugapriyan ${ }^{\mathrm{c}}$, and Lakshmanan $\mathrm{M}^{\mathrm{d}}$ \\ Professor/EEE, Vardhaman College of Engineering, Hyderabad, Telangana, India. \\ BProfessor/EEE , CMR College of Engineering \& Technology, Hyderabad, Telangana, \\ India \\ ${ }^{\mathbf{C}}$ Associate Professor/EEE, Vel Tech Multi Tech Dr. Rangarajan Dr. Sakunthala \\ Engineering College, Avadi, Chennai, India \\ dAssociate Professor/EEE, CMR Institute of Technology, Bengaluru, Karnataka, India.
}

Article History: Received: 11 January 2021; Accepted: 27 February 2021; Published online: 5 April 2021

\begin{abstract}
The necessity of Distributed Generation (DG) in the modern power system has increased greatly. Optimal placement and sizing of DGs have a significant impact on the objectives of voltage stability enhancement, real and reactive power loss minimization and power system security and reliability. Different types of DGs such as Type I, Type II, Type III and Type IVare placed. The optimal placement of these DGs is found using Voltage Stability Index (VSI). The optimal sizing of these DGs is done using Differential Evolution (DE) algorithm. MATLAB simulation is carried out in standard IEEE 33-bus test system. The test is performed for various combinations of different types of DG. The results show that the voltage stability index along with Differential Evolution algorithm provides better voltage profile and power loss minimization as compared to the system without DG.
\end{abstract}

Keywords: Distributed Generation, Voltage Stability Index, Differential Evolution Algorithm, Voltage profile.

\section{Introduction}

Distribution system is an integral part of the power system which supplies electric power to the end consumers. Also, majority of the losses occur in the distribution section of the power system. Due to the growth in industries and increase in the consumption of electrical energy in day-to-day life, the problem of overloading occurs. Voltage stability, increased losses and reduced power quality are the common problems that arise due to overloading [1]. Nowadays, there is a fast transition in the structure of the distribution system. The concept of distributing power from large generating plants through long interconnected transmission networks are subjected to severe issues like transmission loss, voltage stability, network congestion and security [2]. Moreover, increase in renewable energy and the gradual depletion of fossil fuels has led to the emergence of small power generating plants located near the consumer end known as Distributed Generation. Distributed Generation is an active source which supplies energy to the load and is located near to the point of consumption [3]. The sources of DGs are either solar, wind, mini hydro, fuel cell, biomass, geothermal or gas turbine. DGs benefit us in many ways by electricity cost savings, loss reduction, voltage profile improvement, system reliability enhancement, power quality improvement and so on [4]. In today'smodern distribution system, DGs are used to enhance the performance of the system. But, to maximize the benefits of DGs, proper sizing and placement is necessary. Sizing and placement of DGs is done based on different analytical and computational methods [5].

Griffin et al in his work has deployed fuel cell generators throughout the distribution system to reduce power loss and maximize capacity savings [6]. Wang et al has proposed an analytical method to determine the optimal placement of DG with unity power factor in radial as well as networked systemsfor power loss minimization [7].Acharya et al [8] in his work has used sensitivity factor equation to find the optimal placement of DG. Kashem et al has proposed SQP algorithm for finding the optimal location and sizing of DG to reduce power loss at marginal DG cost [9]. Wang and Singh in his work has suggested an Ant Colony System algorithm for the proper allocation of DG based on a reliability index [10]. P. Vijay Babu et al in his work has reported the optimal sitting and sizing of DGs on IEEE 33 bus and IEEE 69 bus using GAMS software and MATLAB. The proposed method was found to be better than the existing one in minimization of power loss and voltage profile improvement [11]. The authors had used dragon fly algorithm to place the DGs on IEEE 15, 33 and 69 bus systems. The proposed algorithm results were compared with other evolutionary algorithms from literature and found to be better [12]. Adel et al have proposed water cycle algorithm to place DGs and Capacitor Banks to improve the voltage profile of the distribution system. The proposed algorithm also improves the emissions from the generation sources [13].

Shanmugapriyan et al has proposed Distributed Generation Suitability Index (DGSI) to find out the placement of DG and sizing is obtained using Differential Evolution and Particle Swarm Optimization algorithms and the results are compared [14]. Muthubalaji et al has proposed an integrated approach to locate DSTATCOM in the distribution system. The authors have used an integrated approach of MACO and BFOA algorithms to locate DSTATCOM in the test systems IEEE 30 and IEEE 69 bus systems [15]. Sedighi et al [16] has used PSO 
algorithm to locate DGs and Alinejad-Beromi et al has used Genetic algorithm [17] for the proper allocation of DGs satisfying the objectives of voltage profile improvement, active power loss, cost minimization and THD minimization. Apart from power loss and cost minimization, maximization of environmental benefits was met through the multi- objective optimization based optimal allocation of DGs proposed by Hong Cui et al[18].

This paper uses voltage stability index for finding optimal DG siting and differential evolution algorithm sizing for satisfying the objectives of enhancing voltage stability and reducing active and reactive power losses. This paper is summarized as Section 1 which describe the importance of DG in the distribution system and gives a glimpse of literature which portrays how the DGs are effectively used to enhance the voltage stability and reliability of the distribution system. Section 2 describes the importance of Voltage Stability Index. Section 3 describe about the problem formulation. Section 4 explains about the optimal placement and sizing of DG. Section 5 explains about the Differential Evolution Algorithm and the procedure to place and size the two types of DG one after the other.

\section{Voltage Stability Index (Vsi)}

To avoid major blackouts, the measure of voltage stability has to be considered. The effective operation of the distribution system is based on its reliability. Hence, while designing a distribution system the factor of voltage stability should be calculated using some specified index. So, there is a need of some index which evaluates the stability so that necessary measures can be taken in case of instability and to improve the voltage stability of the power system. An index which is used to estimate stability is known as Voltage Stability Index (VSI). The closeness of a given operating point to voltage instability can be measured by this tool. The operators use this index for determining the real time operation of the power system. It can be used online or offline. The value of VSI lies between 0 and 1 . VSI is calculated using the formula:

$\mathrm{VSI}=4 \mathrm{X} /\left(\mathrm{V} \_1^{\wedge} 2\right)\left(\left(\mathrm{P} \_2^{\wedge} 2\right) / \mathrm{Q} \_2+\mathrm{Q} \_2\right) \leq 1$

where

$X$ - reactance of the system

$\mathrm{P} 2$ - active power of the load

$\mathrm{Q} 2$ - reactive power of the load and

V_1- source voltage.

\section{Problem Formulation}

The DGs are optimally located and sized with the objective of minimizing Ploss, Qloss and VSI. So, mathematically, these can be formulated as:

To minimize active power loss, Ploss

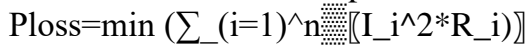

Where

Ii - line current at bus $\mathrm{i}$ in amps and

$\mathrm{R} \_\mathrm{i}$ - the line resistance in ohms.

To minimize reactive power loss, Qloss

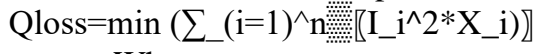

Where

$\mathrm{X} \_\mathrm{i}$ - line reactance at bus $\mathrm{i}$ in ohms.

To minimize Voltage Stability Index,

$\mathrm{VSI}=4 \mathrm{X} /\left(\mathrm{V} \_1^{\wedge} 2\right)\left(\left(\mathrm{P} \_2^{\wedge} 2\right) / \mathrm{Q} \_2+\mathrm{Q} \_2\right) \leq 1$

Constraints

$\mathrm{V} \_$imin $\leq \mathrm{V} \_\mathrm{i} \leq \mathrm{V}$ imax

where $\mathrm{V}_{-} \mathrm{i}$ is the voltage magnitude at bus $\mathrm{i}$;

V_imin- lower limit of voltage

V_imax- upper limit of voltage

$\mathrm{P} \quad$ DGimin $\leq \mathrm{P} \quad$ DGi $\leq \mathrm{P} \quad$ DGimax

where $P_{-}$DGimin - minimum value of $P_{-}$DGi

[ $\mathrm{P}$ ]_DGimax - maximum value of PDGi

Q_DGimin $\leq$ Q_DGi $\leq$ Q_DGimax

where Q_DGimin - minimum value of Q_DGi

Q_DGimax- maximum value of QDGi

\section{Optimal Placing and Sizing of Dg}

Sitting and sizing of DG is important in a distribution system for its proper operation and planning. This is a non-linear constrained optimization problem. This problem is divided into two sub-problems. The first subproblem is to optimally locate the DG such that the voltage stability index is maximized and voltage stability is enhanced. To improve the voltage stability, DG is placed at the weakest bus in the distribution system. The second sub-problem is to optimally size the DG such that the active and reactive power loss minimization is obtained. Here, four types of DG are considered such as:

Type 1: DG which injects active power (P) only. 
Type 2: DG which injects reactive power (Q) only.

Type 3: DG which injects active power $(\mathrm{P})$ and absorbs reactive power $(\mathrm{Q})$.

Type 4: DG injects both active $(\mathrm{P})$ and reactive power $(\mathrm{Q})$.

From the various combinations of the above different types of DGs, the best combination which provides the minimum power loss and better voltage profile is identified.

\section{Differential Evolution Algorithm}

Rainer Storn and Kenneth Price has introduced the optimization algorithm called Differential Evolution algorithm. Its robustness and ease of use has made it popular among the researchers for solving different objective problems. Differential algorithm is being used to determine the sizing of DGs. The number of particles is taken as 20 and the number of iterations is fixed as 50. Optimal location of DG is found out using voltage stability index. The various steps involved are as follows:

1. Run the load flow to obtain base case results.

2. Total active and reactive power loss without DG is determined.

3. Calculate Voltage Stability Index at each node for the test system and arrange them in descending order.

4. Suitable placement of DG is obtained using the lowest value of VSI.

5. Two DGs are to be placed. The first one is placed using the VSI index.

6. Optimize the capacity of DGs using Differential Evolution Algorithm using the objective function equations.

7. For the estimated value of DG capacity, the power loss is calculated.

8. Then the capacity of DG is adjusted in small steps and for each iteration the power loss is determined and is compared with the previous value.

9. The capacity of DG with minimum real and reactive power loss is the finalized sizing of DG.

10. Steps from 3 to 9 is repeated to find the placement and sizing of second DG.

\section{Results and Discussion}

IEEE 33-bus system, having 5 ties and 32 sectionalizing switches, with a total load of 3.7 MW and 2.3 MVAR is taken as the test system and is shown in Fig. 1.

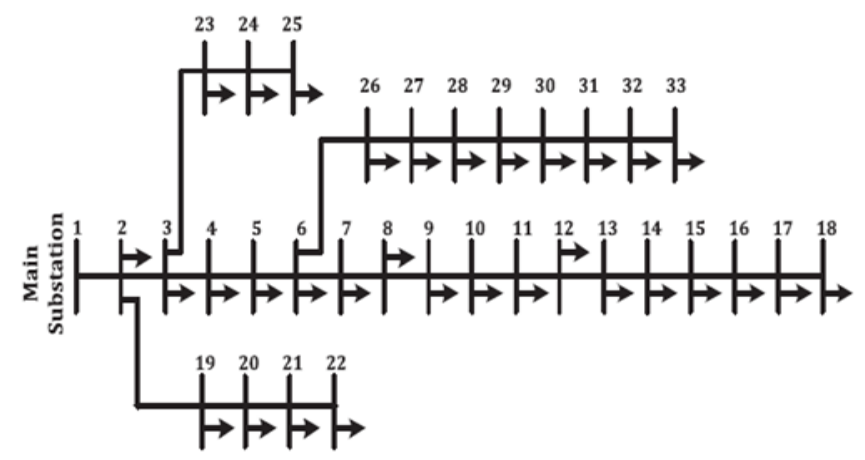

Fig.1 IEEE 33 Bus Test System

Case I: Base case of IEEE 33 Bus Test System

The base case results of IEEE 33-bus system are obtained from the load flow solution. The total active power loss is $210.0594 \mathrm{KW}$ and total reactive power loss is $142.5320 \mathrm{KVAR}$. First the location of first DG is found out. It is obtained by placing $1 \mathrm{KW}$ DG at each bus and VSI for the same is calculated. Then the VSI are arranged in the descending order which gives the weakest bus prone to stability issues. So, the first DG is placed at that location. Then along with the first DG, second DG from various types are placed and its proper placement is found. Once the location of DGs is found, its rating is obtained using DE algorithm. For each combination of DG located at proper placement the active and reactive power losses are found out. The combination with the least active and reactive power loss is considered as the optimum DGs for the performance improvement of the distribution system.

Case II: with Type 1 \& Type 1 DG

In this case, the optimal allocation of type $1 \mathrm{DG}$ is done. Then along with existing type $1 \mathrm{DG}$ another type 1 DG is placed and the active, reactive power loss is found out. The location of both the DGs with loss minimisation is shown in Table 1.

Table 1

Active and reactive power loss after placing two DGs

\begin{tabular}{|c|c|c|c|c|c|c|}
\hline $\begin{array}{c}\text { Sl. } \\
\text { No }\end{array}$ & Type of DG & VSI & DG location & $\begin{array}{c}\text { Rating } \\
\text { KW }\end{array}$ & $\begin{array}{c}\text { Ploss } \\
\text { KW }\end{array}$ & $\begin{array}{c}\text { Qloss } \\
\text { KVAR }\end{array}$ \\
\hline 1 & 1 & 0.1598 & 25 & 0.6463 & 186.2625 & 128.4633 \\
\hline 2 & 1,2 & 0.1440 & 25,24 & $0.6463,0.5240$ & 175.6408 & 122.9854 \\
\hline
\end{tabular}

From the Table 1, it is clear that the rating of Type 1 DG obtained is $0.6463 \mathrm{KW}$ and that of type 2 DG obtained is $0.5240 \mathrm{KW}$. With these two types of DGs placed in the distribution system, first DG in the 25thbus 
and second DG in the 24th bus, the active power loss has been reduced from $210.0594 \mathrm{KW}$ to $175.6408 \mathrm{KW}$. Similarly, the reactive power loss has been reduced from $142.5320 \mathrm{KW}$ to $122.9854 \mathrm{KW}$. Also, the voltage profile of the system is increased as shown in fig. 2.

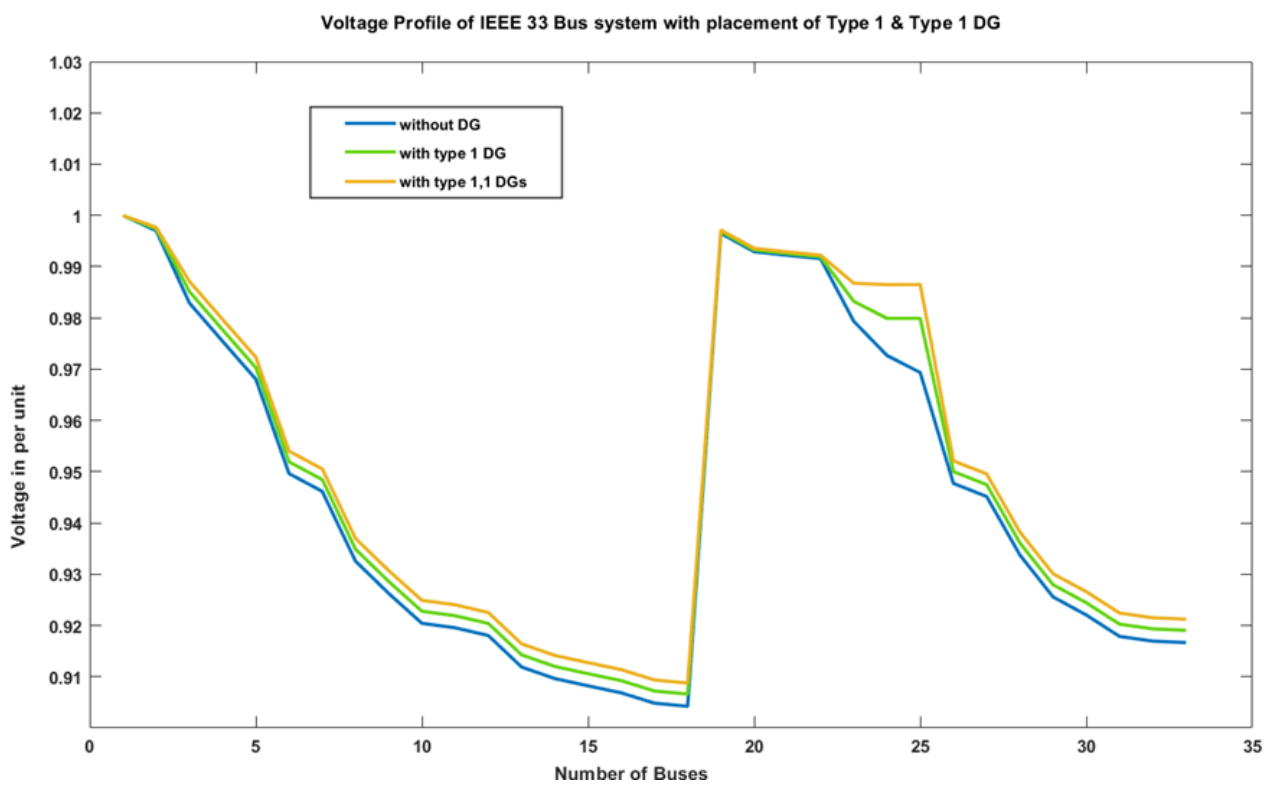

Fig. 2 Voltage profile of the system with type $1 \&$ Type 2 DG

Similarly, three more cases (Type $1 \&$ Type 2 DG, Type $1 \&$ Type 3 DG and Type $1 \&$ Type 4 DG) results are shown in table 2 and their voltage profiles are shown in fig. 3, fig. 4 and fig. 5.

Table 2

Active and reactive power loss after placing two DGs for different cases

\begin{tabular}{|c|c|c|c|c|c|c|}
\hline $\begin{array}{l}\text { Sl. } \\
\text { No }\end{array}$ & $\begin{array}{l}\text { Type of } \\
\text { DG }\end{array}$ & VSI & $\begin{array}{c}\text { DG } \\
\text { location }\end{array}$ & $\begin{array}{l}\text { Rating } \\
\text { KW }\end{array}$ & $\begin{array}{l}\text { Ploss } \\
\text { KW } \\
\end{array}$ & $\begin{array}{l}\text { Qloss } \\
\text { KVAR } \\
\end{array}$ \\
\hline \multirow{2}{*}{2 Case } & 1 & 0.1576 & 25 & 0.5813 & 187.9308 & 129.3643 \\
\hline & 1,2 & 0.1436 & 25,11 & $\begin{array}{c}0.5813, \\
1.2785\end{array}$ & 80.0181 & 55.5258 \\
\hline \multirow{2}{*}{ Case } & 1 & 0.1569 & 25 & 0.5563 & 188.6129 & 129.7398 \\
\hline & 1,3 & 0.1502 & 25,24 & $\begin{array}{c}0.5563, \\
0.5576\end{array}$ & 192.0438 & 132.7890 \\
\hline \multirow{2}{*}{ Case } & 1 & 0.1595 & 25 & 0.6401 & 186.4153 & 128.5447 \\
\hline & 1,4 & 0.1533 & 25,11 & $\begin{array}{l}0.6401, \\
0.9076\end{array}$ & 149.8441 & 105.0414 \\
\hline
\end{tabular}

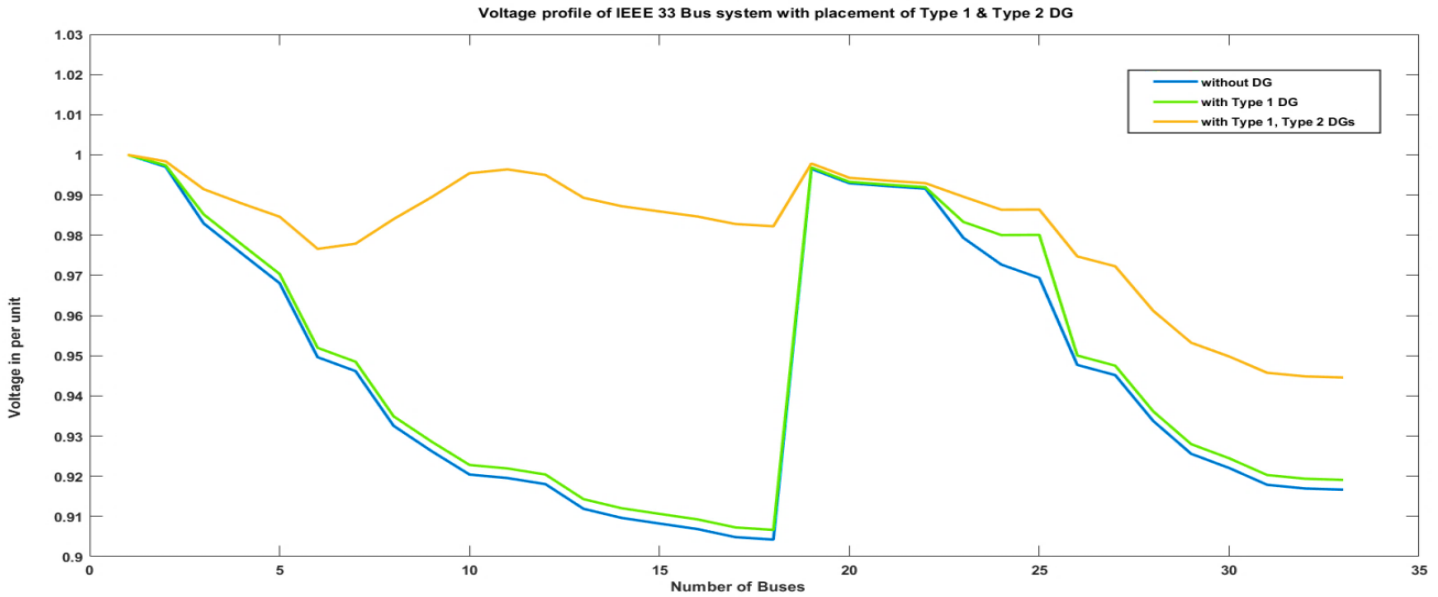

Fig.3 Voltage profile of the system with Type $1 \&$ Type 2 DG 


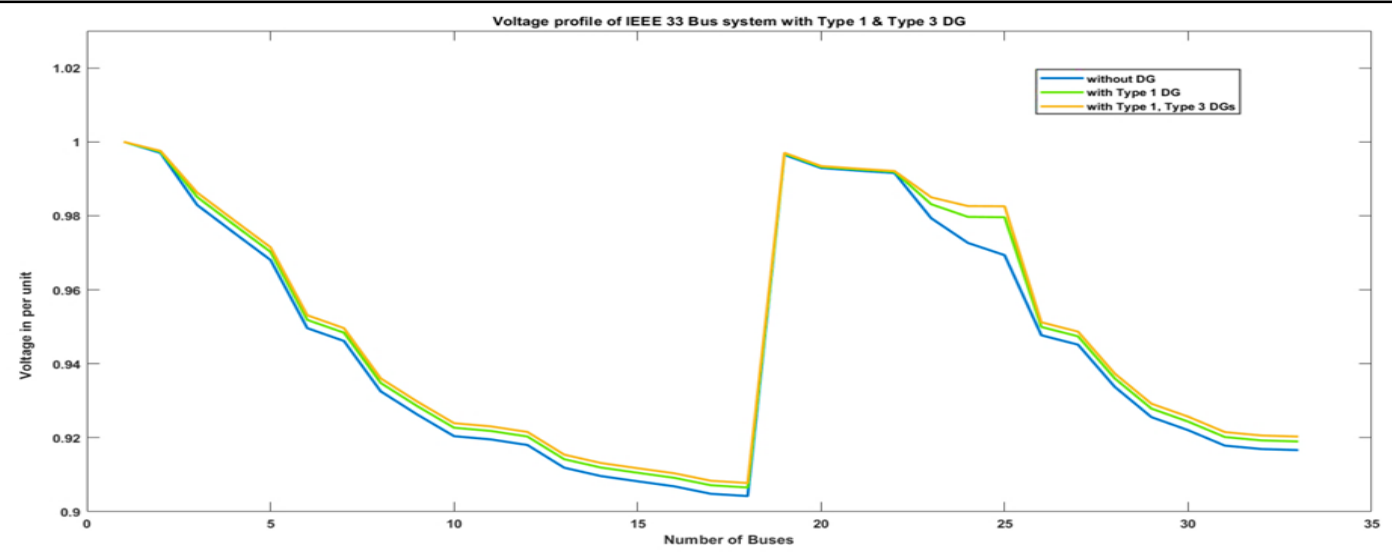

Fig.4 Voltage profile of the system with Type 1 \& Type 3 DG

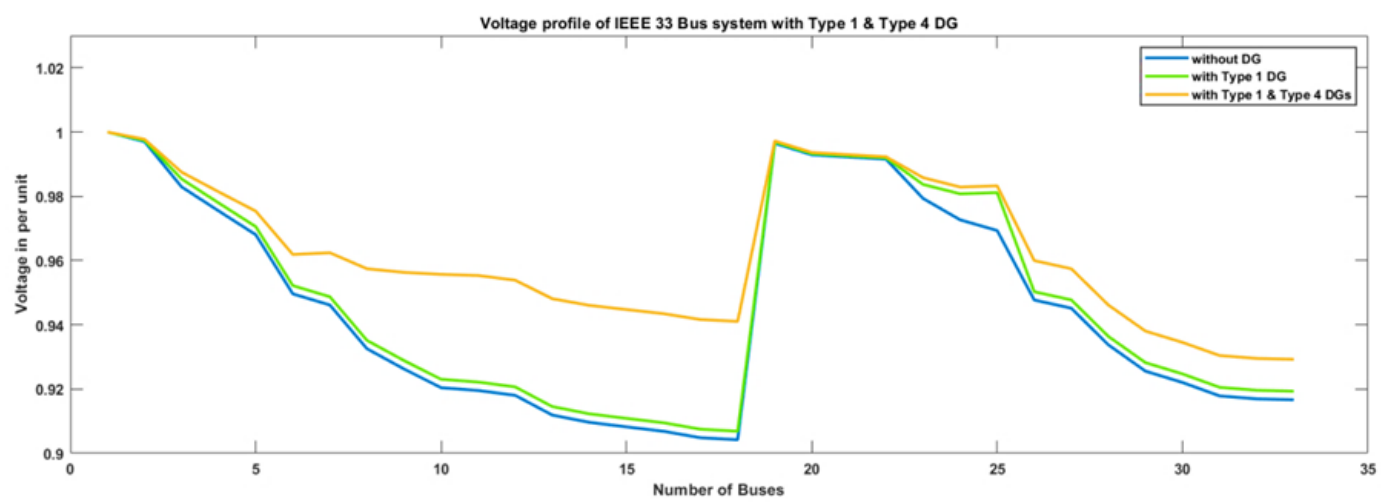

Fig.5 Voltage profile of the system with Type $1 \&$ Type 4 DG

Similarly, the performance evaluation of sixteen combinations of four different types of DG are done and the results are tabulated below:

Table 3

Active and reactive power loss after placing two DGs for different combinations

\begin{tabular}{|c|c|c|c|c|c|}
\hline Sl. No & Combination of DG & DG location & $\begin{array}{c}\text { Rating } \\
\text { KW }\end{array}$ & $\begin{array}{c}\text { Ploss } \\
\text { KW }\end{array}$ & $\begin{array}{c}\text { Qloss } \\
\text { KVAR }\end{array}$ \\
\hline 1 & $1 \& 1$ & 25,24 & $0.6463,0.5240$ & 175.6408 & 122.9854 \\
\hline 2 & $1 \& 2$ & 25,11 & $0.5813,1.2785$ & 80.0181 & 55.5258 \\
\hline 3 & $1 \& 3$ & 25,24 & $0.5563,0.5576$ & 192.0438 & 132.7890 \\
\hline 4 & $1 \& 4$ & 25,11 & $0.6401,0.9076$ & 149.8441 & 105.0414 \\
\hline 5 & $2 \& 1$ & 11,25 & $1.2921,0.6212$ & 79.2322 & 55.1331 \\
\hline 6 & $2 \& 2$ & 11,30 & $1.0945,0.7403$ & 42.7559 & 29.1366 \\
\hline 7 & $2 \& 3$ & 11,25 & $1.3798,0.5472$ & 94.4815 & 65.5306 \\
\hline 8 & $2 \& 4$ & 11,33 & $1.3848,0.7136$ & 71.5624 & 50.8258 \\
\hline 9 & $3 \& 1$ & 25,24 & $0.7833,0.5318$ & 193.2281 & 134.6151 \\
\hline 10 & $3 \& 2$ & 25,11 & $0.8015,1.1404$ & 95.5752 & 65.6501 \\
\hline 11 & $3 \& 3$ & 25,24 & $0.7039,0.5350$ & 216.0978 & 148.7596 \\
\hline 12 & $3 \& 4$ & 25,11 & $0.6514,1.0494$ & 164.5201 & 115.6494 \\
\hline 13 & $4 \& 1$ & 11,25 & $0.9697,0.6318$ & 150.1490 & 105.5093 \\
\hline 14 & $4 \& 2$ & 11,30 & $0.8816,0.9801$ & 81.9983 & 58.6367 \\
\hline 15 & $4 \& 3$ & 11,25 & $0.9079,0.8426$ & 160.9242 & 113.1122 \\
\hline 16 & $4 \& 4$ & 11.33 & $0.9148,0.7723$ & 148.4765 & 103.8713 \\
\hline
\end{tabular}

It is clear from table 3 that the 6th combination, i.e., the combination of type 2 and type 2 , with the first type 2 DG at 11 th bus and the second type 2 DG at 30thbus results in active power loss of $42.76 \mathrm{KW}$ and reactive 
power loss of $29.13 \mathrm{KW}$. This combination improves the performance of the distribution system by minimizing the active and reactive power loss.

\section{Conclusion}

In this work, the Voltage Stability Index (VSI) determines the optimal location of DG and optimal size of DG is found using differential evolution algorithm. The DG is located at the weakest bus which is determined by the VSI and the DG is sized considering the active and reactive power losses using Differential Evolution Algorithm. The methodology is tested for sixteen combinations of different types of DG and the combination which results in minimum active and reactive power loss is determined. In the future scope, the cost of DG and the placement of DG using other sensitivity indexes can be considered.

\section{References}

1. Sankaramoorthy Muthubalaji, Veluchamy Malathi, "Multi-objective distribution feeder reconfiguration by considering Energy not supplied with distributed generation”, vol. 22, no. 6, pp. 1539-1545, 2015.

2. N. Karuppiah, V. Malathi, "Damping of Power System Oscillations by tuning of PSS and SVC using Particle Swarm Optimization”, Tehnicki Vjesnik - Technical Gazette, vol. 23, no. 1, pp. 221-227, 2016.

3. Karuppiah Natarajan, Muthubalaji Sankaramoorthy, Anand Rajkumar, "Controlling the Power Loss in Radial Distribution Network by Optimally placing UPQC through reconfiguration using Evolutionary Algorithms", International Journal of Pure and Applied Mathematics, Vol. 118, no. 16, pp. 161-179, 2018.

4. Muthubalaji S., Malathi V. (2015) Optimal Loss Reduction and Reconfiguration of Distribution System with Distributed Generation Using Harmony Search Algorithm. In: Panigrahi B., Suganthan P., Das S. (eds) Swarm, Evolutionary, and Memetic Computing. SEMCCO 2014. Lecture Notes in Computer Science, vol 8947. Springer, Cham.

5. S. Muthubalaji, N. Karuppiah, R. Anand, "Performance Improvement of Distribution System by Optimal Placement and Sizing of Distributed Energy Storage Systems and DGS Using Particle Swarm Optimization Algorithm", Journal of Advanced Research in Dynamical and Control systems, vol. 10, special issue 14, pp. 864-871, 2018.

A. Law, K. Tomsovic, D. Secrest and T. Griffin, "Placement of Dispersed Generations Systems for Reduced Losses," in Proceedings of the 33rd Hawaii International Conference on System Sciences - 2000, pp. 1-9.

6. Caisheng Wang and M. H. Nehrir, "Analytical approaches for optimal placement of distributed generation sources in power systems," in IEEE Transactions on Power Systems, vol. 19, no. 4, pp. 20682076, Nov. 2004.

7. Naresh, Acharya, PukarMahat, N.Mithulananthan, "An analytical approach for DG allocation in primary distribution networkInternational Journal of Electrical Power \& Energy Systems, vol. 28, no. 10, pp. 669-678, 2006.

A. D. T. Le, M. A. Kashem, M. Negnevitsky and G. Ledwich, "Optimal Distributed Generation Parameters for Reducing Losses with Economic Consideration, " 2007 IEEE Power Engineering Society General Meeting, Tampa, FL, USA, 2007, pp. 1-8.

8. Lingfeng Wang and Chanan Singh, "Reliability-Constrained Optimum Placement of Reclosers and Distributed Generators in Distribution Networks Using an Ant Colony System Algorithm”, IEEE transactions on systems, man, and cybernetics-part c: applications and reviews, vol. 38, no. 6, November 2008.

9. P. Vijay Babu, S.P. Singh, "Optimal Placement of DG in Distribution Network for Power Loss Minimization Using NLP \& PLS Technique”,Energy Procedia, Volume 90,2016, Pages 441-454.

10. Suresh, M.C.V., Belwin, E.J. Optimal DG placement for benefit maximization in distribution networks by using Dragonfly algorithm. Renewables 5, 4 (2018)

A. A. A. El-Ela, R. A. El-Sehiemy and A. S. Abbas, "Optimal Placement and Sizing of Distributed Generation and Capacitor Banks in Distribution Systems Using Water Cycle Algorithm," in IEEE Systems Journal, vol. 12, no. 4, pp. 3629-3636, Dec. 2018.

11. J. Shanmugapriyan, N. Karuppiah, S. Muthubalaji and S. Tamilselvi, "Optimum placement of multi type $D G$ units for loss reduction in a radial distribution system considering the distributed generation suitability index using evolutionary algorithms", Bulletin of the Polish Academy of Sciences Technical Sciences, Vol. 66, No. 3, 2018

12. S. Muthubalaji, R. Anand, N. Karuppiah, "An Integrated Optimization approach to locate the DSTATCOM in power distribution system to reduce the power loss and total cost”, Periodicals of Engineering and Natural Sciences, vol. 6, no. 2, pp. 283-294, 2018.

13. M. Sedighi, A. Igderi and A. Parastar, "Sitting and sizing of Distributed Generation in distribution network to improve of several parameters by PSO algorithm," 2010 Conference Proceedings IPEC, Singapore, 2010, pp. 1083-1087. 
N. Karuppiah ${ }^{a}$, S. Muthubalaji ${ }^{b}$, J.Shanmugapriyan ${ }^{c}$, and Lakshmanan $M^{d}$

14. Y. Alinejad-Beromi, M. Sedighizadeh, M. R. Bayat and M. E. Khodayar, "Using genetic algorithm for distributed generation allocation to reduce losses and improve voltage profile," 2007 42nd International Universities Power Engineering Conference, Brighton, UK, 2007, pp. 954-959.

15. H. Cui and W. Dai, "Multi-objective optimal allocation of distributed generation in smart grid," 2011 International Conference on Electrical and Control Engineering, Yichang, China, 2011, pp. 713-717. 